\section{Extensive Collaboration on Large-Scale Projects}

A Joint Appeal by the Presidents of The American Physical Society (APS) and the European Physical Society (EPS)

As a result of a joint meeting of the Presidents and other executives of the EPS and the APS in Budapest on 25-27 May 1992, we jointly issue the following appeal:

Physics research relies more and more on important large facilities or big instruments, each serving a large number of users. These tools are often required in research pursuits that strive to extend the limits of our knowledge.

The size, complexity and cost of these facilities, and the growing number of users which each of them serves, calls for increasing international collaboration. We believe that more cooperation and even collaboration should be sought.

Therefore, the APS and EPS urge that international collaboration in the choice, design, construction, exploitation, and possibly even in the site selection, of future very large physics facilities should be developed to the fullest extent possible in order to use the funds for research in the most cost-effective way.

A collaboration procedure, which is becoming a requirement for even medium-scale projects in Europe, should include the United States for very large projects. Eventually, such collaborations should extend to the whole industrialized world.

Both societies stand ready to offer assistance in the organization of forums, workshops, topical conferences, and communication through their respective Divisions, publications and networks. They will pool their information and act in concert whenever appropriate.

In addition, the two societies will make all effort so that access to large facilities is open, for all qualified users, irrespective of their geographical origin.

E. Henley, M. Jacob

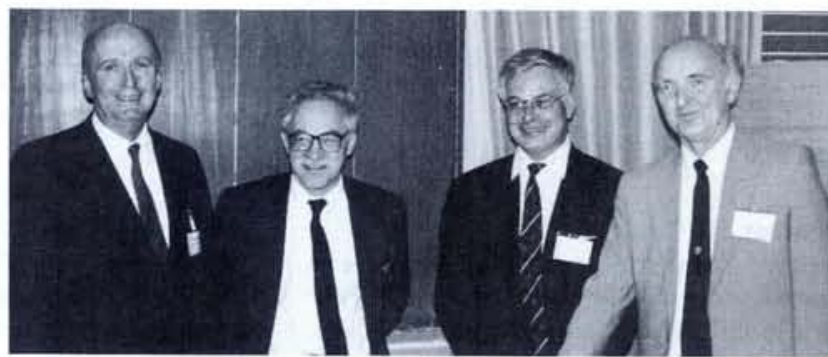

From left to right: Donald Langenberg, President-Elect of The American Physical Society (APS) and Chancellor of the University of Maryland System; Ernest Henley, APS President, of the University of Washington who acted as the first Director of the new institute for nuclear theory in Seattle; Maurice Jacob, EPS President; and Norbert Kroo, EPS Vice-President.

\section{Condensed Matter Division Board CALL FOR NOMINATIONS}

Members of the EPS Condensed Matter Division are invited to submit by the end of September, 1992, the names of candidates for election to the Divisional Board. The names should be sent to G. Thomas, the Executive Secretary, at the EPS Secretariat, Nádor u. 7, H-1051 Budapest.

Three supporting signatures of Individual Members of EPS are required as well as the written agreement of the candidate that he or she is willing to serve. The term of office is three years and re-election for a second period is allowed. The Board includes the Section Committee Chairmen and five members are elected by members of the Division. Four of the Board will shortly complete their terms of office (E. Hilfrich, V. Smid, J.B. Sousa, and M. Surma) and have served for two successive three-year terms so cannot stand for re-election. J. Devreese (Antwerp) is willing to be a candidate for a second term.

\title{
FORSCHUNGSZENTRUMJÜLICH GmbH
}

We are a national research centre jointly financed by the Federal Republic of Germany and the federal state of North Rhine-Westphalia with a staff of approx. 4500. The five major research priorities comprising structure of materials/ materials research, information technology, energy technology, environmental research and life sciences are part of a broad spectrum of basic research ranging from medicine and biotechnology to solid-state research and basic nuclear research.

At our INSTITUTE OF THIN FILM AND ION TECHNOLOGY there is a vacancy for a scientist (male or female) as the

\section{Director (C4) (successor to Prof. Dr. Ch. Heiden)}

to head the DEPARTMENT OF SUPERCONDUCTOR ELECTRONICS AND TECHNOLOGY.

Research and development work at the Institute of Thin Film and lon Technology (ISI) is an integral part of the KFA's research priority on "Basic Research on Information Technology". In two departments, the Institute of Superconductor Electronics and Technology and the Institute of Semiconductor Electronics and Technology, research is carried out on the deposition, structuring and modification of layers and film systems for the two material classes of superconductors and semiconductors, and, on the basis of these findings, novel devices are developed. In accordance with the staff and apparatus available, major priority is given to work on basic principles and application of the specific properties of ceramic high-temperature superconductors for micro- and cryoelectronics. The combination of semiconducting and superconducting components for hybrid devices is also of interest.

Applications are invited from scientists with an appropriate qualification and extensive experience in the research areas described above. Candidates should be capable of heading a large institute operating on an interdisciplinary basis and should be prepared to cooperate with industry, other institutes at the KFA, and universities at home and abroad.

In a joint procedure together with a university in the Federal State of North Rhine-Westphalia, the Director will be appointed to a university chair and then seconded to take over the appointment at the KFA. The salary will conform to the C4 scale of the German Civil Service; "Habilitation" or equivalent scientific qualifications are required.

Applications accompanied by a curriculum vitae, list of publications and a brief description of previous scientific activities should be submitted by September 30, 1992 to the

Postfach 1913, 5170 Jülich, Federal Republic of Germany 